\title{
Soil moisture retrieval from multi-incidence angle observations at L-band
}

\author{
$\underline{\text { S. Peischl }}{ }^{\text {a }}$, N. Ye ${ }^{\text {a }}$, J.P. Walker ${ }^{\mathrm{a}}$, D. Ryu ${ }^{\mathrm{b}}$ and Y.H. Kerr \\ ${ }^{a}$ Department of Civil Engineering, Monash University, Australia \\ ${ }^{2}$ Department of Infrastructure Engineering, The University of Melbourne, Australia \\ ${ }^{c}$ Centre d'Etudes Spatiales de la Biosphère (CESBIO), Toulouse, France \\ (E-mail: sandy.peischl@monash.edu)
}

\begin{abstract}
Soil moisture is an important environmental variable in regulating energy fluxes and water infiltration near the soil surface. This makes it a significant parameter in meteorological and climate modelling applications. While ground measurement of soil moisture at a large spatial scale is cumbersome and time-consuming, remote sensing offers the advantage of frequent observations in time and space. The most promising remote sensing technique for soil moisture observations is microwave radiometry at L-band, which is highly sensitive to moisture and less affected by roughness and canopy attenuation than compared to shorter wavelengths in the microwave range. Consequently, the first dedicated mission for global Soil Moisture and Ocean Salinity mapping (SMOS), launched in November 2009, has a passive microwave radiometer at $\mathrm{L}$-band $(1.4 \mathrm{GHz})$ that uses a two-dimensional interferometric $\mathrm{Y}$-shaped antenna.
\end{abstract}

The novel design of this satellite, yielding multi-incidence angle observations, provides a unique opportunity to acquire accurate information on near surface soil moisture. However, the newly developed algorithms for this mission need to be thoroughly tested on a wide range of land surface conditions and spatial resolutions, since their parameterization has been mostly limited to small scale field studies that focused on data from tower radiometers and simulation experiments. The SMOS mission is based on the relationship between the measured brightness temperature and the dielectric constant of the soil, which is related to its moisture content. Since this relationship is affected by a range of factors, including surface roughness and vegetation cover, the SMOS mission is relying on the multi-incidence angle observations to estimate some of the ancillary parameters required by the retrieval algorithm.

In order to assess the performance of the core algorithm used by SMOS, multi-angle airborne data at L-band were studied from an Australian field campaign in 2005 (NAFE - National Airborne Field Experiment). The flights were conducted across three focus areas capturing a range of vegetation and soil moisture conditions on several observation days. The airborne instrument operated was the Polarimetric L-band Multi-beam Radiometer (PLMR) which provided dual-polarized brightness temperature measurements at six different incidence angles. The multi-angle observations were obtained by deploying a push-broom sensor rotated such that all beams were looking along the flight track, three forward and three backward, respectively. Corresponding ground sampling activities at specific focus farms included near surface-soil moisture, profile soil temperature, vegetation temperature, vegetation water content and biomass measurements. Additional rainfall, soil moisture profile and soil temperature data were collected at permanent monitoring sites nearby.

In this research the L-MEB model was used to investigate the soil moisture retrieval results obtained when only specific ranges of multi-angle brightness temperature observations over wheat canopy were used. Moreover, the impact of varying moisture conditions and multi-parameter retrievals were studied to further assess the model performance and soil moisture accuracy. The results demonstrated overall good soil moisture estimates in relation to the ground measurements $\left(\Delta \mathrm{SM}=0-0.03 \mathrm{~m}^{3} / \mathrm{m}^{3}\right)$, if i) soil moisture was retrieved solely and ii) all available brightness temperature data collected across $0-50^{\circ}$ incidence angles were used. However when attempting the simultaneous retrieval of soil moisture together with ancillary data and focusing on specific angular ranges of observations, large variations in soil moisture estimates were observed. In particular, L-band data collected at incidence angles of $10^{\circ}$ or higher seemed to be more strongly affected by the canopy, since vegetation effects of the dominant vertical wheat structure increased with larger incidence angles. Hence, L-band data measured at near-nadir views $\left(0-10^{\circ}\right)$ produced the soil moisture results closest to those measured - independently of the observed moisture conditions - when the optical depth and/or the surface roughness parameter were retrieved simultaneously with soil moisture.

Keywords: $L-M E B$, microwave radiometry, multi-incidence angle, $N A F E$, remote sensing, SMOS 


\section{INTRODUCTION}

The retrieval of soil moisture from L-band microwave observations over vegetated areas is relatively complex compared to bare soil conditions, since the additional vegetation contribution on the emission signal needs to be accounted for within the model. Thus, with the increasing number of input parameters, even more ground information is needed to ensure accurate soil moisture estimates from remote sensing instruments, such as the recently launched Soil Moisture and Ocean Salinity (SMOS) satellite (Kerr et al., 2010a). The ancillary data might be obtained from different ground and space borne instruments. However, issues due to spatial or temporal discrepancies between the different data sources arise. Consequently, previous work has studied the use of dual-polarized multi-incidence angle L-band data to retrieve soil moisture simultaneously with some of the ancillary data needed as model input (Wigneron et al., 2000). It was shown that these socalled multi-parameter retrievals using the multi-angle capability of sensors such as that deployed by SMOS may enhance the overall retrieval process, depending on the field of view, the moisture conditions, the vegetation type and growth state. However, if a radiometer instrument is only capable of brightness temperature acquisition at a very narrow range of incidence angles compared to SMOS $\left(\sim 0-60^{\circ}\right)$, the accuracy of soil moisture estimated from multi-parameter retrieval might change depending on the angular range chosen for multi-angle observations; e.g. the near nadir measurements of SMOS are strongly affected by the low antenna gain in that particular area of the field of view. Hence, this paper analyses the multiangular soil moisture retrieval quality over a wheat canopy site using airborne brightness temperature data collected at different ranges of incidence angles. The effect of i) changing moisture conditions and ii) the type and number of simultaneously retrieved parameters on the model performance are also investigated.

\section{EXPERIMENTAL DATA}

The data used in this paper was sourced from the National Airborne Field Experiment (NAFE'05), conducted during October-November 2005 in South-East Australia (Panciera et al., 2008). The campaign focused on the northern part of the Goulburn River catchment $\left(32^{\circ} \mathrm{S}, 150^{\circ}\right.$ E) located in New South Wales (Figure 1). Within the main study region of $40 \times 40 \mathrm{~km}$, several smaller focus areas were selected for intensive airborne and ground operations at farm scale. The experimental data set comprised among others, airborne multiincidence angle L-band measurements together with ground information from extensive in-situ sampling and monitoring stations. The Merriwa Park farm (Figure 1), which was the focus of this paper, had a gently sloping topography and was characterized by a mixture of wheat and grass land use on a silty clay loam soil.

\subsection{Airborne Data}

The airborne microwave radiometer used in the NAFE'05 campaign was the Polarimetric L-band Multi-beam Radiometer (PLMR), which is capable of dual-polarized brightness temperature measurements (TB) at a frequency of $1.413 \mathrm{GHz}$ and bandwidth of $24 \mathrm{MHz}$. The instrument is equipped with six receiving beams, with fixed look angles of $\pm 7^{\circ}, \pm 21.5^{\circ}$ and $\pm 38.5^{\circ}$. For the multi-angle flights, PLMR was mounted in along-track configuration on the aircraft. This provided three beams pointing forward and three beams backward with respect to the flight direction of the aircraft. Considering an aircraft pitch of approximately $4^{\circ}$, the resulting look angles of the six PLMR beams were typically $3^{\circ}, 11^{\circ}, 17^{\circ}, 26^{\circ}, 34^{\circ}$ and $43^{\circ}$ in along-track mode. Consequently, as the aircraft moved along its flight path at a nominal altitude of $750 \mathrm{~m}$, quasisimultaneous multi-angle measurements at approximately $250 \mathrm{~m}$ spatial resolution were made for the same location on earth. The multi-angle data were acquired in the early afternoon between 12:00 pm and 3:00 pm on a total of four observation days (2-Nov, 9-Nov, 16-Nov and 23-Nov). The PLMR instrument was calibrated before and after each flight using cold (sky) and warm (blackbody box) calibration targets. Additional in-flight calibration checks were conducted through low-altitude flights across a large water body, which was monitored for surface water temperature and salinity. The instrument accuracy is given as $0.7 \mathrm{~K}$ for $\mathrm{H}$ - and $2 \mathrm{~K}$ for $\mathrm{V}$-polarization (Panciera et al., 2008). Further processing of the multi-angle data included

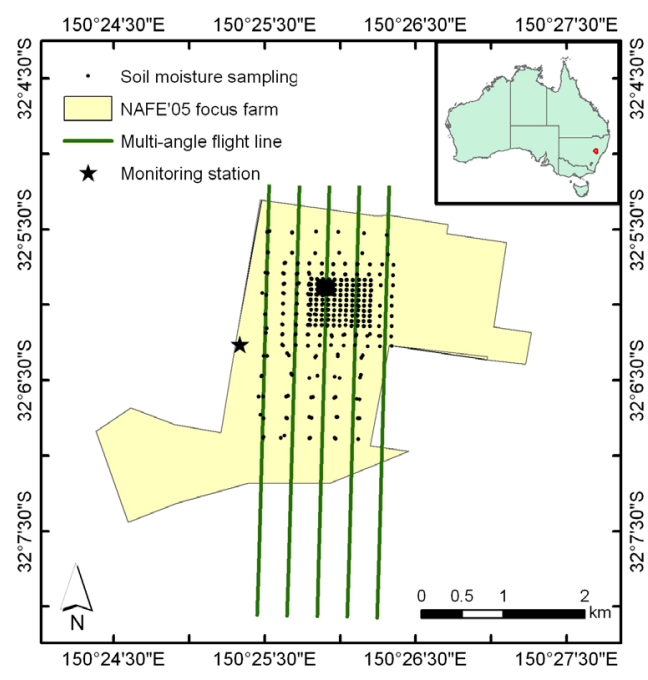

Figure 1. Outline of the Merriwa Park focus farm with the aircraft flight lines, the ground soil moisture sampling grid $(6.25 \mathrm{~m}-2 \mathrm{~km})$

and the location of monitoring stations overlain. Inset: Location of the NAFE'05 test site in Australia. 
calculation of the effective footprint size and the local incidence angle, taking into account the surface topography, aircraft position and attitude. Moreover, some of the PLMR data with large yaw and/or roll angles caused by strong cross-wind and turbulence were filtered.

\subsection{Ground Data}

Extensive ground sampling was conducted at the focus farm within an area of $1.5 \times 3.0 \mathrm{~km}^{2}$, concurrent to the airborne observations. The near-surface $(0-5 \mathrm{~cm})$ soil moisture measurements $(\mathrm{SM})$ were made with the Hydraprobe Data Acquisition System (HDAS), which includes a Hydraprobe soil moisture sensor, a Global Positioning System (GPS) and a handheld pocket PC (Merlin et al., 2007). The calibration of the Hydraprobe standard soil moisture product was based on gravimetric soil samples and laboratory data. Considering the correction of temperature effects, the estimated accuracy of the HDAS system is $\pm 0.033 \mathrm{~m}^{3} / \mathrm{m}^{3}$ (Merlin et al., 2007). During the campaign, surface moisture contents were measured between 9:00 am to 1:30 pm on a regular grid with varying resolution. The spacing between the individual sampling points ranged from $6.25 \mathrm{~m}$ to $2 \mathrm{~km}$ and was designed in such a way that the high-resolution sampling zone was located within the cropping area of the Merriwa Park focus farm (Figure 1). Supplementary data including surface roughness, vegetation type, dew amount, vegetation water content and biomass were also collected by the ground teams. Long-term profile soil moisture $(0-5 \mathrm{~cm}, 0-30 \mathrm{~cm}, 30-60 \mathrm{~cm}$ and $60-90 \mathrm{~cm})$, soil temperature $(0-5 \mathrm{~cm}$ and $0-$ $30 \mathrm{~cm}$ ) and rainfall records were available from an existing in-situ monitoring network (Rüdiger et al., 2007). Moreover, temporary monitoring stations were set up during the period of the campaign and equipped with thermal infrared sensors (TIR), surface soil temperature instrumentation $(1 \mathrm{~cm}, 2.5 \mathrm{~cm}$ and $4 \mathrm{~cm})$ and leaf wetness sensors. The overall dynamic range of surface soil moisture measured was between $0.14-0.46 \mathrm{~m}^{3} / \mathrm{m}^{3}$ at Merriwa Park. In response to a significant rainfall event before the start of the campaign, substantial drying effects of the topsoil were observed throughout the field experiment.

\section{RADIOMETRIC MODEL}

The core component of the operational soil moisture retrieval algorithm for SMOS is the L-band Microwave Emission of the Biosphere model (L-MEB), explained in detail in Wigneron et al. (2007) and implemented into the Community Microwave Emission Modelling Platform (CMEM)(Drusch et al., 2009). This radiative transfer model is a simplified (zero-order) solution to describe the $p$-polarized ground emission $\left(e_{G P}\right)$ taking into account the presence of an overlying vegetation layer (with $p=H$ for horizontal and $p=V$ for vertical polarization). The contribution of the canopy to the soil reflectivity $\left(r_{G P}=1-e_{G P}\right)$ is modelled in terms of the vegetation attenuation $\left(\gamma_{P}\right)$ and scattering effects $\left(\omega_{P}\right)$, which together result in a composite brightness temperature $\left(T B_{P}\right)[\mathrm{K}]$. Considering the soil and vegetation components and the effective temperature $[\mathrm{K}]$ of both media, i.e. soil $T_{G}$ and vegetation $T_{C}$, the so-called tau-omega model is given as (Mo et al., 1982)

$$
T B_{P}=\left(1-\omega_{P}\right)\left(1-\gamma_{P}\right)\left(1+\gamma_{P} r_{G P}\right) \cdot T_{C}+\left(1-r_{G P}\right) \gamma_{P} \cdot T_{G}
$$

The reflectivity of the soil surface can be estimated by the Fresnel reflectivity $\left(r_{G P}^{*}\right)$ and adjusted for nonsmooth surfaces through the use of additional parameters, such as $H_{R}$ and $N_{R P}$ (Wang and Choudhury, 1981):

$$
r_{G P}=r_{G P}^{*} \cdot \exp \left[-H_{R} \cos \theta^{N_{R P}}\right] .
$$

The $H_{R}$ parameter is included as a semi-empirical effective surface roughness parameter and $N_{R P}$ for the dependency of the surface roughness on the incidence angle $(\theta)$. When modelling of the canopy attenuation, further referred to as transmissivity, the angular effect on the signal needs also to be taken into account through the optical depth $\left(\tau_{P}\right)$ by

$$
\gamma_{P}=\exp \left[-\tau_{P} / \cos \theta\right] \text {. }
$$

The optical depth as shown in (3) is strictly valid for nadir-views only and can be approximated using a linear function of the vegetation water content $(V W C)\left[\mathrm{kg} / \mathrm{m}^{2}\right]$ and the empirical parameter $b_{P}$. The latter is mainly dependent on sensor frequency, polarization, canopy type and plant structure (Jackson and Schmugge, 1991):

$$
\tau_{N A D}=V W C \cdot b_{P} .
$$

Consequently, to correct for non-nadir observations two additional vegetation structure parameters $t t_{P}$ are introduced, that account for an increasing $\left(t t_{P}>1\right)$ or decreasing $\left(t t_{P}<1\right)$ trend in the optical depth and hence in the vegetation transmissivity as a function of the incidence angle. For the particular case of $t t_{P}=1$, the $\tau_{P}$ of the standing canopy is independent of polarization and incidence angle, which is defined as isotropic state.

$$
\tau_{P}=\tau_{N A D}\left(\sin ^{2} \theta \cdot t t_{P}+\cos ^{2} \theta\right) .
$$


For the purpose of this study, the L-MEB model was used to solve an optimization problem between the airborne dual-polarized brightness temperatures and the forward simulated brightness temperatures by iteratively minimizing a cost-function through least-square to retrieve soil moisture and ancillary data. Consequently, the number of parameters that were retrieved simultaneously depended on the number of available brightness temperature measurements for the same location on earth.

\section{MULTI-INCIDENCE ANGLE SOIL MOISTURE RETRIEVAL}

In order to investigate how a specific angular range of brightness temperature measurements performs for soil moisture retrieval, all available L-band data within a designated PLMR pixel over the Merriwa Park wheat area were categorized according to their incidence angle information (Table 1). Note that due to the configuration of the PLMR instrument, there were a few cases where groups classified at a finer angular scale did not contain any data, but have been included for the sake of completeness. The total number of $T B_{p}$ studied (collected across incidence angles of $0-50^{\circ}$ ) depended on the individual observation day, but ranged from 15 observations on the $16^{\text {th }}$ November 2005 to $27-32$ observations for the three remaining days. The corresponding HDAS measurements for the particular day/pixel comprised about $250-300$ points, which were averaged and the spatial variability estimated from the standard deviation.

The soil moisture retrieval was classified into single-parameter retrieval (soil moisture) and multi-parameter retrieval, considering - apart from soil moisture (SM) - additional model parameters as unknown. These ancillary parameters included the surface roughness $H_{R}$ and the vegetation water content $V W C$. The vegetation water content was calculated from the retrieved optical depth information using Equation 4 . The semi-empirical roughness parameter $H_{R}$ was set as either dependent on the retrieved soil moisture $H_{R}=f(\mathrm{SM})$ or independent within the retrieval process. The concept of a soil moisture dependent $H_{R}$ parameter is also applied in the processing of SMOS data (Kerr et al., 2010b), since work by Escorihuela et al. (2007) indicated better retrieval results when taking into account the topsoil moisture content for determining $H_{R}$. With regard to the NAFE'05 data set, similar results were found for the study site and the different moisture conditions observed (Peischl et al., 2011; Panciera et al., 2009a; 2009b). Recent findings suggest that the soil water contribution in $H_{R}$ might alternatively result from a difference in sampling depth between the L-band radiometer observations and the ground measurements (Escorihuela et al., 2010). The remaining L-MEB model parameters including soil texture, soil temperature, vegetation temperature, vegetation scattering albedo and further surface roughness characterization were taken mostly from literature (Wigneron et al., 2007) or available in-situ measurements. The parameterization used in this study had been previously confirmed and tested for the same test site (Peischl et al., 2011), demonstrating maximum root mean square errors of 2-5 K. The soil moisture retrievals were performed i) on all four multi-angle flight days over the Merriwa Park cropping area and ii) for each individual group of angular brightness temperature measurements across the different scenarios (Table 1).

\subsection{Single-parameter Retrieval}

The single-parameter retrieval (1-P) focused solely on retrieving soil moisture and assumed all remaining LMEB model input data as known. The resulting soil moisture estimates for each group of brightness temperatures (Table 1) are displayed in Figure 2. Generally, using all available angular brightness temperature observations for the 1-P soil moisture retrieval produced values close to the ground measurements for all observation days $\left(\Delta \mathrm{SM}=0-0.03 \mathrm{~m}^{3} / \mathrm{m}^{3}\right)$. However by focusing on brightness temperatures collected at specific incidence angle ranges, the simulation accuracy varied. In particular, $T B_{p}$ associated with very large incidence angles $\left(>40^{\circ}\right)$ tended to slightly overestimate soil moisture for very wet and very dry conditions. In addition, for soil moisture conditions less than $0.22 \mathrm{~m}^{3} / \mathrm{m}^{3}$, consistently lower values compared to the ground information were retrieved when only $T B_{p}$ data at small incidence angles were used $\left(<15^{\circ}\right)$. Overall, all grouped soil moisture retrievals were still within the range of the standard deviation of the ground measurements independent of the varying moisture conditions observed.

Table 1. Overview of grouping criteria for PLMR brightness temperature measurements according to their corresponding incidence angle information.

\begin{tabular}{|c|ccccccccc|c|}
\hline \multirow{2}{*}{ Scenario } & \multicolumn{1}{|c|}{ Group Identifier for Incidence Angle Ranges [ ${ }^{\circ}$ ] } \\
& $\mathbf{1}$ & $\mathbf{2}$ & $\mathbf{3}$ & $\mathbf{4}$ & $\mathbf{5}$ & $\mathbf{6}$ & $\mathbf{7}$ & $\mathbf{8}$ & $\mathbf{9}$ \\
\hline $\mathbf{A}$ & $0-50$ & & & & & & & & \\
B & $0-25$ & $25-50$ & & & & & & & \\
C & $0-15$ & $15-30$ & $30-50$ & & & & & & \\
D & $0-14$ & $14-22$ & $22-35$ & $35-50$ & & & & & \\
E & $0-10$ & $10-20$ & $20-30$ & $30-40$ & $40-50$ & & & & \\
F & $0-8$ & $8-16$ & $16-24$ & $24-32$ & $32-40$ & $40-50$ & & & \\
G & $0-7$ & $7-14$ & $14-21$ & $21-28$ & $28-35$ & $35-42$ & $42-50$ & & \\
H & $0-5$ & $5-10$ & $10-15$ & $15-20$ & $20-25$ & $25-30$ & $30-35$ & $35-40$ & $40-50$ \\
\hline
\end{tabular}




\subsection{Multi-parameter Retrieval}

The multi-parameter retrieval included three different combinations: i) simultaneous retrieval of soil moisture and vegetation water content; ii) simultaneous retrieval of soil moisture and the surface roughness parameter, and iii) simultaneous retrieval of soil moisture, vegetation water content and surface roughness. All model results are displayed in Figure 2 with the individual retrieval methods differentiated by symbol types. The 2Parameter (2-P) retrieval of soil moisture and $V W C$ showed for most observation days a reasonable agreement between measured and simulated soil moisture, when all available $T B_{p}$ data were used $\left(\triangle \mathrm{SM}=0.02-0.08 \mathrm{~m}^{3} / \mathrm{m}^{3}\right)$. However, focusing on data collected at mid-range and in particular at high incidence angles decreased the soil moisture accuracy significantly $\left(\Delta \mathrm{SM}=0.06-0.38 \mathrm{~m}^{3} / \mathrm{m}^{3}\right)$. In contrast, very good retrieval results were obtained from $T B_{p}$ observations at very small angles close to nadir-view (0$5^{\circ}$ ), implicating less impact by the vegetation due to i) shorter path lengths through the canopy and ii) the effective composite temperature being closer to that of the soil. Further, for very dry soils and a canopy with low vegetation water content $\left(<1 \mathrm{~kg} / \mathrm{m}^{2}\right)$ the vegetation contribution seemed to be less pronounced, enabling soil moisture estimates close to the ground measurements even for brightness temperature observations at angles of $\leq 25^{\circ}$.

The 2-P retrieval of soil moisture and $H_{R}$ independently demonstrated a consistent improvement compared to the 1-P approach across all angular ranges, except for the case of low moisture content. The retrieved values followed exactly the angular behaviour of 1-P, which exhibited minor variations and corresponded well to the measurements in wet conditions. The soil moisture estimates for the last observation day, where the measured moisture was approximately $0.14 \mathrm{~m}^{3} / \mathrm{m}^{3}$, demonstrated a notable angular trend with increasing discrepancies between model and ground data, the larger the incidence angles of the brightness temperature
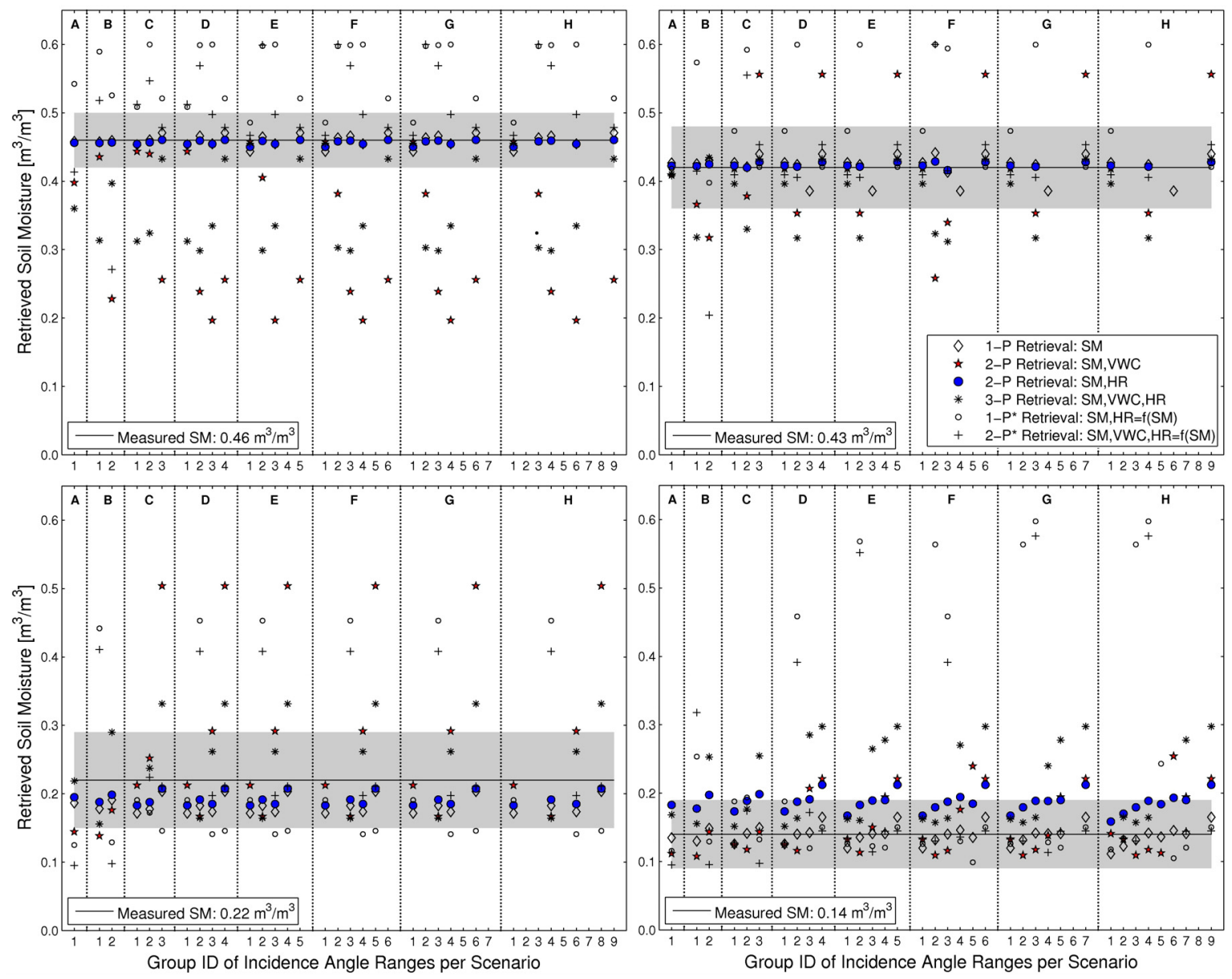

Figure 2. Comparison of modelled moisture values retrieved from airborne brightness temperature data at different incidence angles with measured ground soil moisture given as black line and its standard deviation indicated in grey. Refer to Table 1 for the angular information corresponding to each group and scenario displayed (scenarios are separated by vertical lines). The classification into n-parameter retrievals was based on both the number and type of parameters that were simultaneously derived with soil moisture $(*$ in the legend indicates $H_{R}$ retrieved as a function of SM). 
observations were. This phenomenon might be induced by the increased angular dependency of the optical depth with larger angles causing the model to overcompensate for soil moisture by $\sim 0.05 \mathrm{~m}^{3} / \mathrm{m}^{3}$. With respect to the soil moisture retrieval together with $H_{R}$, but constraining the surface roughness value as dependent on the retrieved soil moisture value, a linear relationship proposed by Panciera et al. (2009a) was implemented into the L-MEB model. Hence, the retrieval was not regarded as an independent 2-P retrieval and instead rather considered as a 1-parameter retrieval $\left(1-\mathrm{P}^{*}\right)$. The modelled soil moisture data were quite different to that estimated by the independent SM- $H_{R}$ approach, and did not follow the angular variations of the 1-P retrieval at all. One feature was particularly evident: that soil moisture values retrieved for the incidence angle range of $15-20^{\circ}$ were far higher than observed $\left(\Delta \mathrm{SM}=0.14-0.46 \mathrm{~m}^{3} / \mathrm{m}^{3}\right)$ across all observations days.

For the simultaneous 3-P retrieval of soil moisture, $V W C$ and $H_{R}$ was characterized by a strong variability of soil moisture estimates across the angles and observation days (Figure 2). In general, best soil moisture retrieval results were obtained from brightness temperature data measured at very small incidence angles $(0$ $5^{\circ}$ ). This supports the earlier hypothesis of reduced signal interference by the canopy layer for near-nadir views. Besides, according to the observed ground conditions, the retrieved soil moisture values tended to underestimate the wet conditions and overestimate the dry conditions, with most of the results being outside the range of the standard deviation given per day. In contrast, the simultaneous 2-P* retrieval of soil moisture, $V W C$ and the soil moisture dependent $H_{R}$, mainly yielded soil moisture values within the range of the standard deviation, which varied slightly across the angular ranges. In comparison with all other retrieval approaches, the minor angular variations were similar to that of the 1-P retrieval of soil moisture with no significant changes for varying moisture conditions.

In order to further assess the performance of the different retrieval approaches across the various incidence angles, the root mean square error (RMSE) between the observed and simulated brightness temperatures were compared for scenario H (Figure 3). Generally, the RMSE tended to decrease with increasing incidence angles for wet conditions, whereas for dry conditions there was little variation across the angles. Moreover, the RMSE calculated for the different n-parameter retrievals applied per observation day were very similar and did not demonstrate any of the discrepancies observed when the actual soil moisture estimates were compared (as shown in Figure 2). This aspect emphasises that the RMSE should not be used as a single criterion for the evaluation of the soil moisture retrieval approach.

\section{CONCLUSION}

This study investigated the impact of using airborne L-band brightness temperature measurements collected at specific ranges of incidence angles on the accuracy of predicted soil moisture estimates at farm scale. Moreover, multi-parameter retrievals and varying moisture conditions were assessed to find the most suitable angular range of observations for soil moisture retrieval over wheat canopy. In general, the best soil moisture estimates in comparison to ground data were modelled from brightness temperature data collected at incidence-angles between $0-5^{\circ}$ among the overall multi-incidence angle range tested $(0-$ $50^{\circ}$ ). This might be related to the dominant vertical structure of the wheat canopy, which affected the soil emission and brightness temperature measurements at near-nadir view to a lesser extent than at large incidence angles, where longer path lengths of the emission through the vegetation layer are more likely to lead to stronger attenuation effects. Additionally, in the case of multi-parameter retrievals model predictions degraded where ancillary data about the vegetation $(V W C)$ was unknown. In contrast, good results with only minor angular variations were observed when soil moisture and surface roughness were retrieved simultaneously. The changing moisture conditions did not show significant impact on the retrieval, except for very dry conditions where the contribution of the vegetation was reduced and instead the surface roughness component (and possibly an increased sampling depth) gained more weight

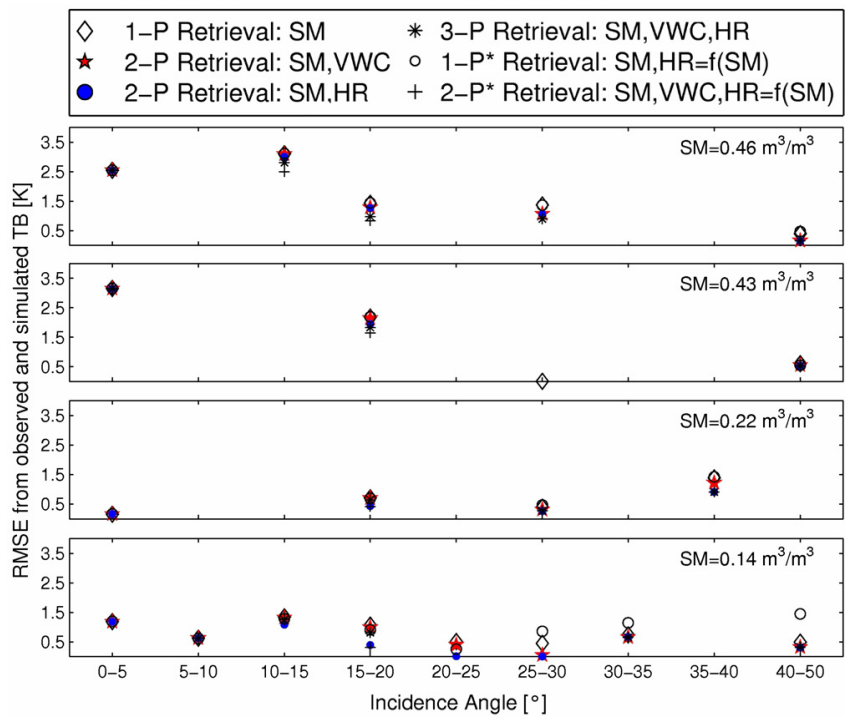

Figure 3. Calculated root mean square error (RMSE) between measured and simulated brightness temperatures (TB) derived for scenario H (Table 1) and n-parameter retrievals considering varying soil moisture conditions. 
in the retrieval algorithm. Since the angular retrieval seemed to be closely linked to the vegetation structure, future work should extend the analysis to a wider range of land use types at varying spatial scales and consider combinations of different angular ranges. Moreover, the retrieval of further ancillary L-MEB parameters, such as the soil or vegetation temperature, should be investigated in particular with regard to SMOS data and the usage of descending orbits, where the vegetation temperature is most likely different to the effective temperature of the soil media.

\section{ACKNOWLEDGMENTS}

The authors would like to thank the NAFE'05 team for providing the data set used in this study and the anonymous reviewers for their helpful comments. This work was supported by the Australian Research Council (DP0879212) as part of the Moisture Map project.

\section{REFERENCES}

Drusch, M., T.R.H. Holmes, P. de Rosnay and G. Balsamo (2009). Comparing ERA-40-based L-band brightness temperatures with Skylab observations: A calibration/validation study using the community microwave emission model. Journal of Hydrometeorology, 10(1), 213-226.

Escorihuela, M.J., Y.H. Kerr, P. de Rosnay, J.-P. Wigneron, J.-C. Calvet and F. Lemaitre (2007). A Simple Model of the Bare Soil Microwave Emission at L-Band. IEEE Transactions on Geoscience and Remote Sensing, 45(7 Part 1), 1978-1987.

Escorihuela, M.J., A. Chanzy, J.-P. Wigneron and Y.H. Kerr (2010). Effective soil moisture sampling depth of L-band radiometry: A case study. Remote Sensing of Environment, 114(5), 995-1001.

Jackson, T.J. and T.J. Schmugge (1991). Vegetation effects on the microwave emission of soils. Remote Sensing of Environment, 36(3), 203-212.

Kerr, Y.H., P. Waldteufel, J.-P. Wigneron, S. Delwart, F. Cabot, J. Boutin, M.J. Escorihuela, J. Font, N. Reul and C. Gruhier (2010a). The SMOS Mission: New Tool for Monitoring Key Elements of the Global Water Cycle. IEEE Transactions on Geoscience and Remote Sensing, 98(5), 666-687.

Kerr, Y.H., P. Waldteufel, P. Richaume, J.-P. Wigneron, P. Ferrazzoli and R.J. Gurney (2010b). SMOS level 2 processor for soil moisture - Algorithm Theoretical Based Document (ATBD), Issue 3.e, pp.121.

Merlin, O., J.P. Walker, R. Panciera, R. Young, J. Kalma and E.J. Kim (2007). Soil moisture measurement in heterogeneous terrain. International Congress on Modelling and Simulation (MODSIM), Modelling and Simulation Society of Australia and New Zealand, New Zealand, December 2007, 2604-2610.

Mo, T., B.J. Choudhury, T.J. Schmugge, J.R. Wang and T.J. Jackson (1982). A model for microwave emission from vegetation-covered fields. Journal of Geophysical Research, 87, 11229-11237.

Panciera, R., J.P. Walker, J.D. Kalma, E.J. Kim, J.M. Hacker, O. Merlin, M. Berger and N. Skou (2008). The NAFE'05/CoSMOS Data Set: Toward SMOS Soil Moisture Retrieval, Downscaling, and Assimilation. IEEE Transactions on Geoscience and Remote Sensing, 46(3), 736-745.

Panciera, R., J.P. Walker, J. Kalma, E.J. Kim, K. Saleh and J.-P. Wigneron (2009a). Evaluation of the SMOS L-MEB passive microwave soil moisture retrieval algorithm. Remote Sensing of Environment, 113(2), 435-444.

Panciera, R., J.P. Walker and O. Merlin (2009b). Improved Understanding of Soil Surface Roughness Parameterization for L-Band Passive Microwave Soil Moisture Retrieval. IEEE Transactions on Geoscience and Remote Sensing, 6(4), 625-629.

Peischl, S., J.P. Walker, D. Ryu, Y.H. Kerr, R. Panciera and C. Rüdiger (2011). Wheat canopy structure and surface roughness effects on multi-angle observations at L-band. IEEE Transactions on Geoscience and Remote Sensing, accepted.

Rüdiger, C., G. Hancock, H.M. Hemakumara, B. Jacobs, J.D. Kalma, C. Martinez, M. Thyer, J.P. Walker, T. Wells and G.R. Willgoose (2007). Goulburn River experimental catchment data set. Water Resources Research, 43(10), W10403.

Wang, J.R. and B.J. Choudhury (1981). Remote Sensing of Soil Moisture Content Over Bare Field at 1.4 GHz Frequency. Journal of Geophysical Research, 86(C6), 5277-5282.

Wigneron, J.-P., P. Waldteufel, A. Chanzy, J.-C. Calvet and Y.H. Kerr (2000). Two-dimensional microwave interferometer retrieval capabilities over land surfaces (SMOS Mission). Remote Sensing of Environment, 73(3), 270-282.

Wigneron, J.-P., Y.H. Kerr, P. Waldteufel, K. Saleh, M.J. Escorihuela, P. Richaume, P. Ferrazzoli, P. de Rosnay, R.J. Gurney, J.-C. Calvet, J.P. Grant, M. Guglielmetti, B. Hornbuckle, C. Mätzler, T. Pellarin and M. Schwank (2007). L-band Microwave Emission of the Biosphere (L-MEB) Model: Description and calibration against experimental data sets over crop fields. Remote Sensing of Environment, 107(4), 639-655. 